\title{
Synthesis of Luminescent Eu(III)-doped Octacalcium Phosphate Particles Hybridized with Succinate Ion and Their Reactive Behavior in Simulated Body Fluid
}

\author{
Iori Yamada, 1, 2 Daichi Noda, ${ }^{1}$ Kenji Shinozaki, ${ }^{3,4}$ \\ Tania Guadalupe Peñaflor Galindo, ${ }^{5}$ Motohiro Tagaya ${ }^{1, ~ *}$ \\ 1 Department of Materials Science and Technology, Nagaoka University of Technology, \\ 1603-1 Kamitomioka, Nagaoka, Niigata 940-2188, Japan \\ 2 Research Fellow of the Japan Society for the Promotion of Science (DC), Tokyo, Japan \\ 3 National Institute of Advanced Industrial Science and Technology (AIST), 1-8-31 \\ Midorigaoka, Ikeda, Osaka 563-8577, Japan \\ 4 PRESTO, Japan Science and Technology Agency (JST), \\ Kawaguchi, Saitama 332-0012, Japan
}

5 General Department, National Institute of Technology, Nagaoka College,

888 Nishikatagai, Nagaoka, Niigata 940-8532, Japan

\footnotetext{
* Author to whom correspondence should be addressed:

Tel: +81-258-47-9345; E-mail: tagaya@mst.nagaokaut.ac.jp
} 


\section{Scheme S1}<smiles>O=C(O)CCC(=O)[18F]</smiles>

$37 \%$<smiles></smiles>

$59 \%$<smiles>O=C([O-])CCC(=O)[O-]</smiles>

$4 \%$

Scheme S1. Illustration of the Suc molecular dissociation structures in water at the $\mathrm{pH}$ value of 4.4 where Suc-OCP:Eu was synthesized. Here, the existence ratios were shown in the figure. 
Figure S1
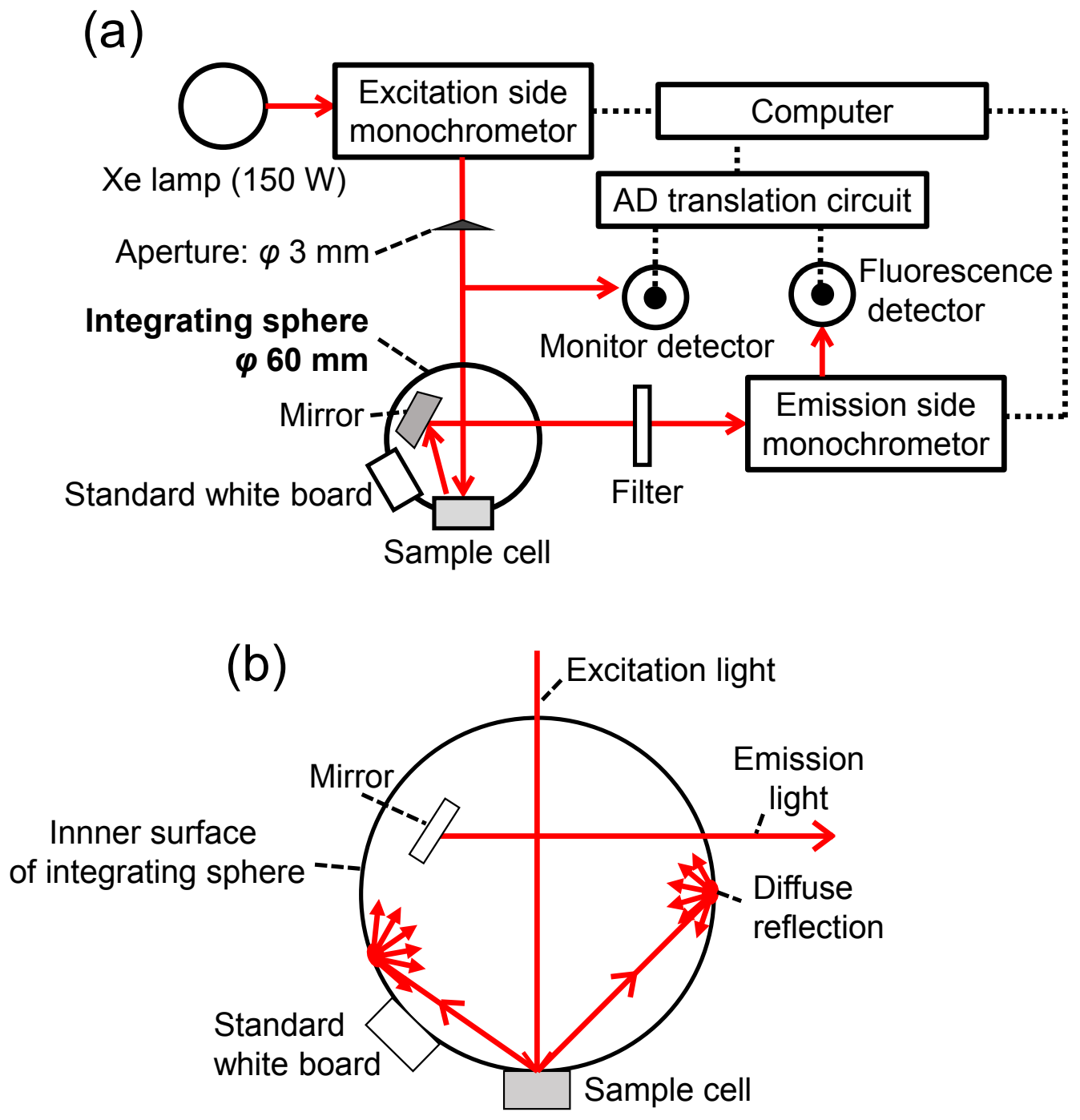

Figure S1. Schematic set-up diagrams of (a) optical system for measuring PL and (b) light pass way inside the integrating sphere. 
Figure 52

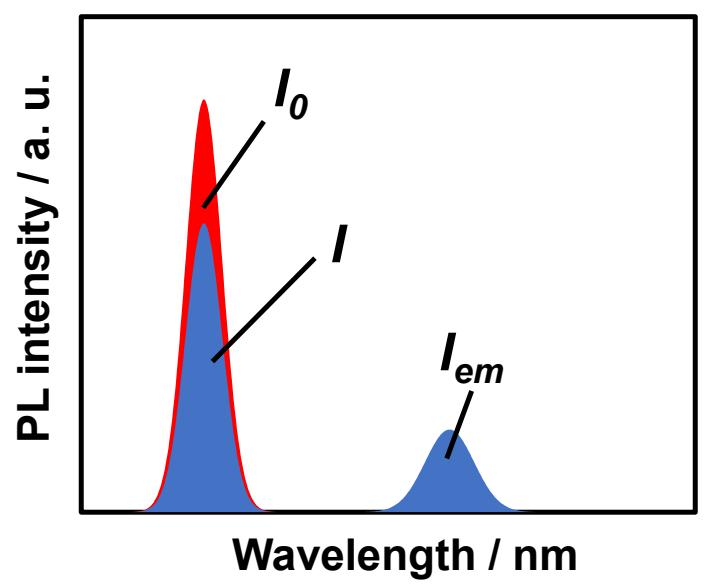

Figure S2. Scheme of the incident, scattering, and luminescence spectra for calculating the $\eta_{\text {int }}$ internal quantum efficiency. The integrated peak intensities attributed to the incident, scattering, and luminescence were abbreviated as $I_{0}, I$ and $I_{\text {em }}$, respectively. 
Figure S3
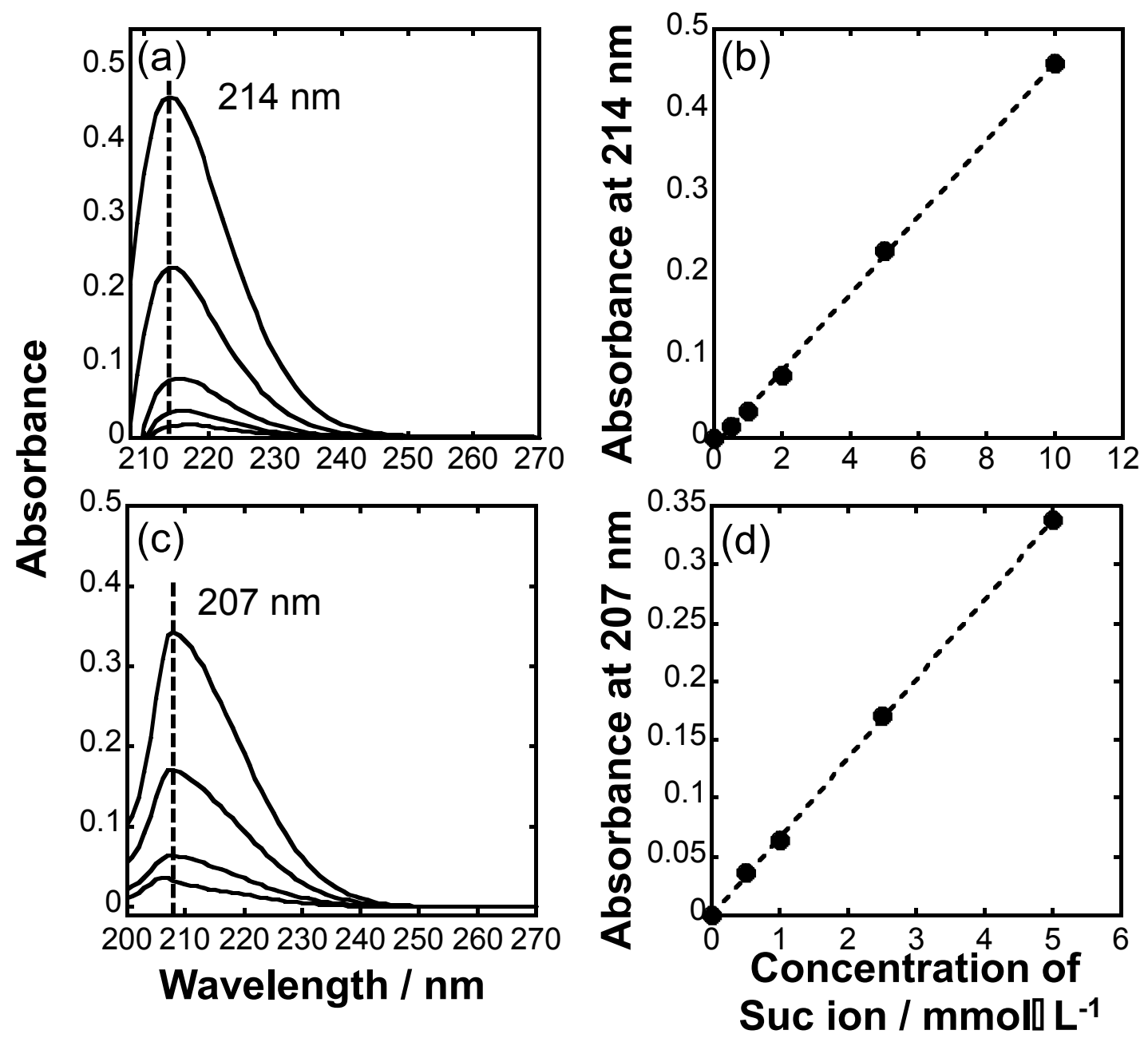

Figure S3. (a, c) UV-Vis absorption spectra and (b, d) calibration curves of Suc ion in (b) SBF ( $R$ $=0.9995)$ and $(\mathrm{d}) \mathrm{HCl}(1 \mathrm{~mol} / \mathrm{L})(R=0.9999)$. 


\section{Figure S4}
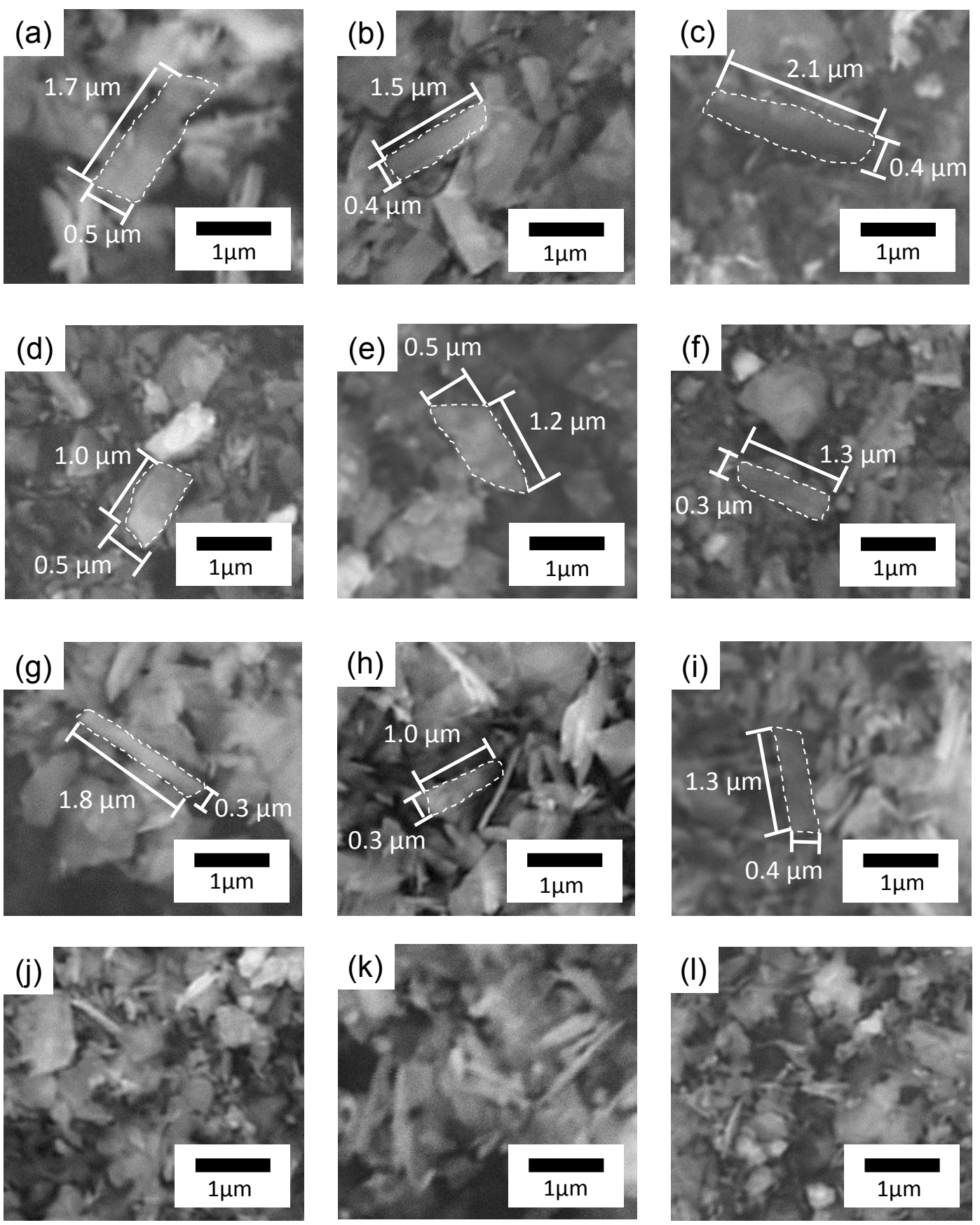

Figure S4. SEM images of (a) OCP:Eu-0, (b) OCP:Eu-1, (c) OCP:Eu-3, (d) OCP:Eu-5, (e) OCP:Eu-7 and (f) OCP:Eu-10, and (g) Suc-OCP:Eu-0, (h) Suc-OCP:Eu-1, (i) Suc-OCP:Eu-3, (j) Suc-OCP:Eu-5, (k) Suc-OCP:Eu-7 and (I) Suc-OCP:Eu-10. 
Figure S5

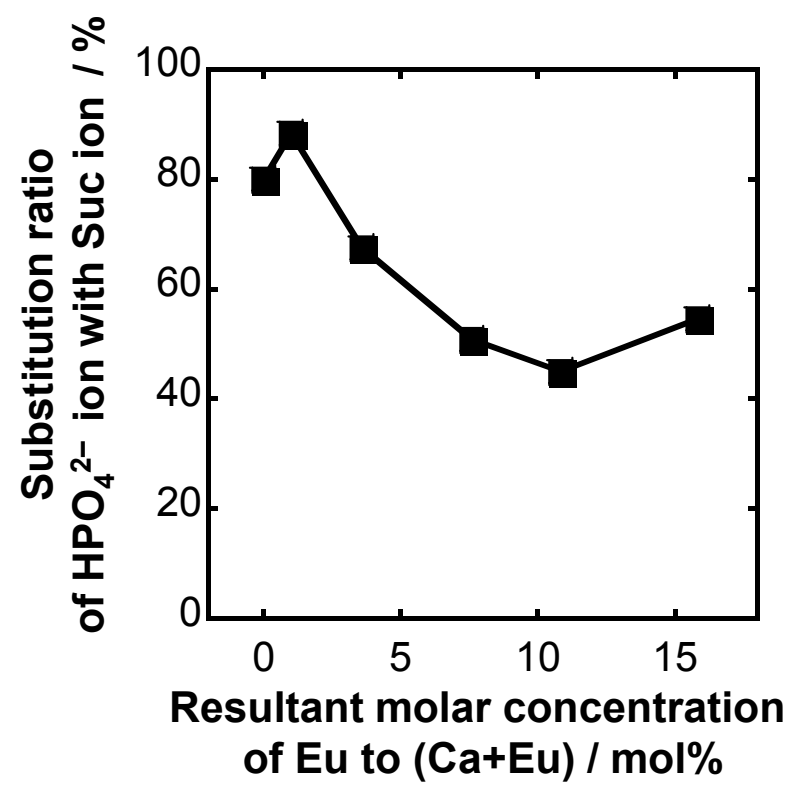

Figure S5. Substitution ratio change of $\mathrm{HPO}_{4}{ }^{2-}$ ion with Suc ion in Suc-OCP:Eu- $\boldsymbol{X}(\boldsymbol{X}=\mathbf{0}, \mathbf{1}, \mathbf{3}, \mathbf{5}$, 7 and 10) with the resultant molar concentration of Eu to (Ca+Eu). 
Figure S6

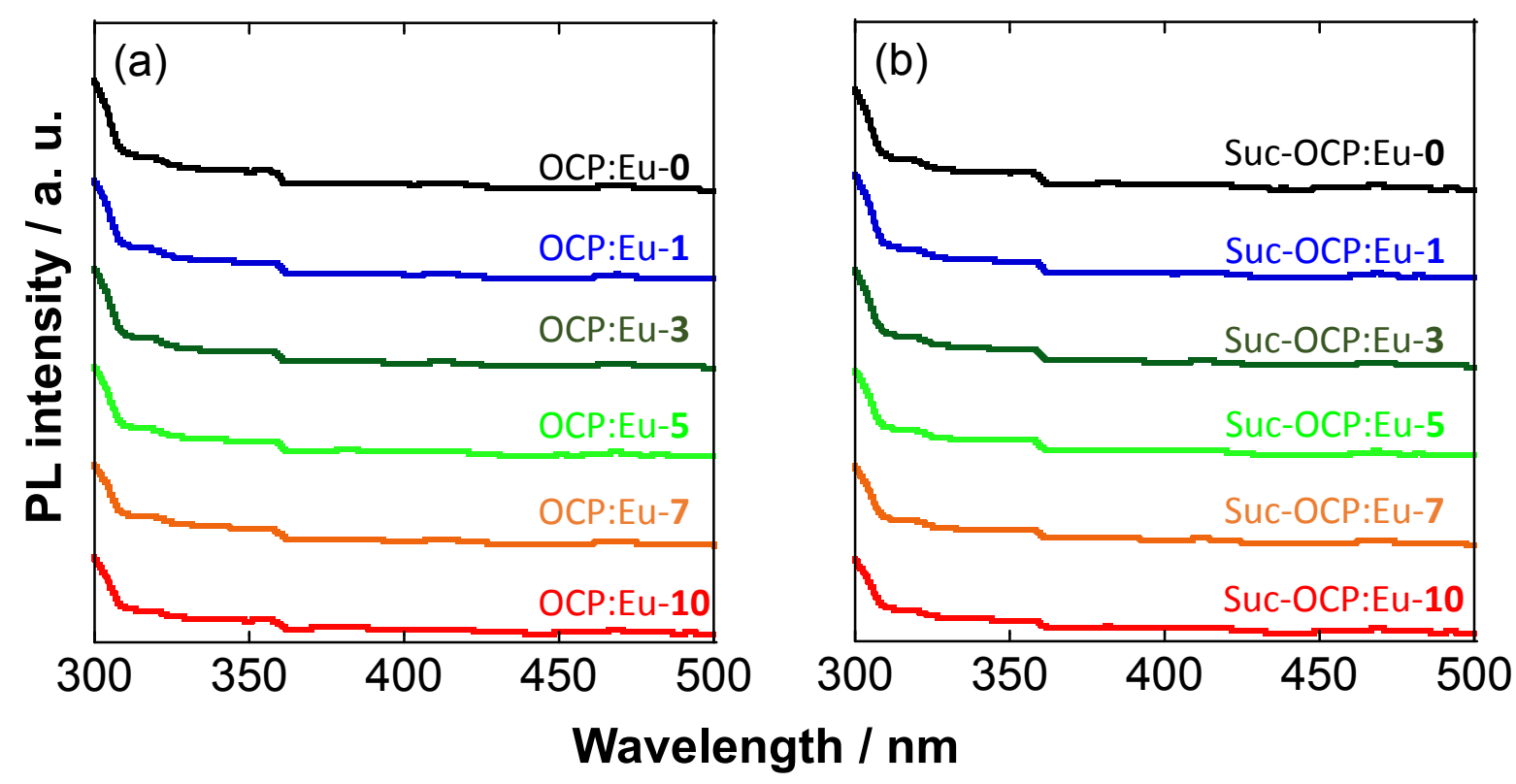

Figure S6. Luminescence spectra of (a) OCP:Eu- $\boldsymbol{X}$ and (b) Suc-OCP:Eu- $\boldsymbol{X}(\boldsymbol{X}=\mathbf{1}, \mathbf{3}, 5,7$ and 10) under the excitation wavelength at $266 \mathrm{~nm}$. 


\section{Figure S7}

(a)
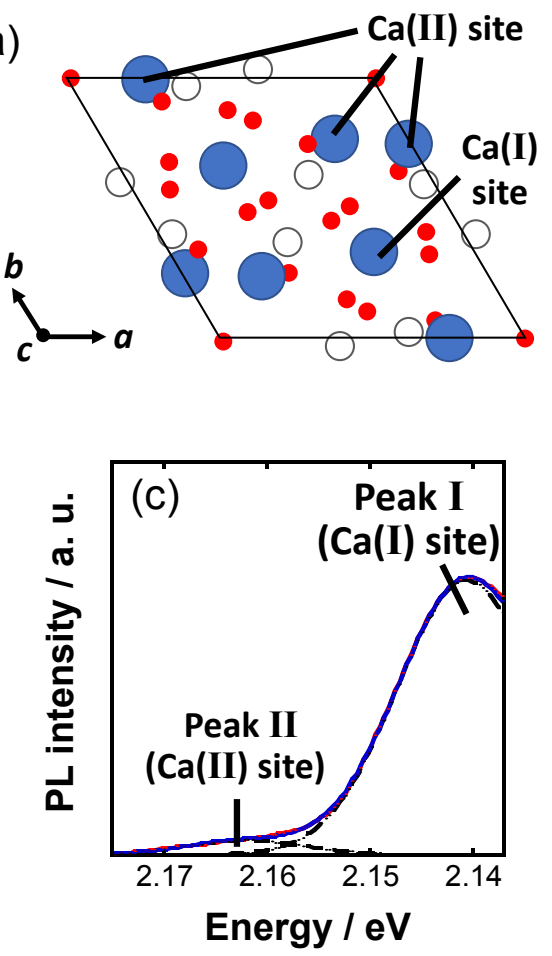
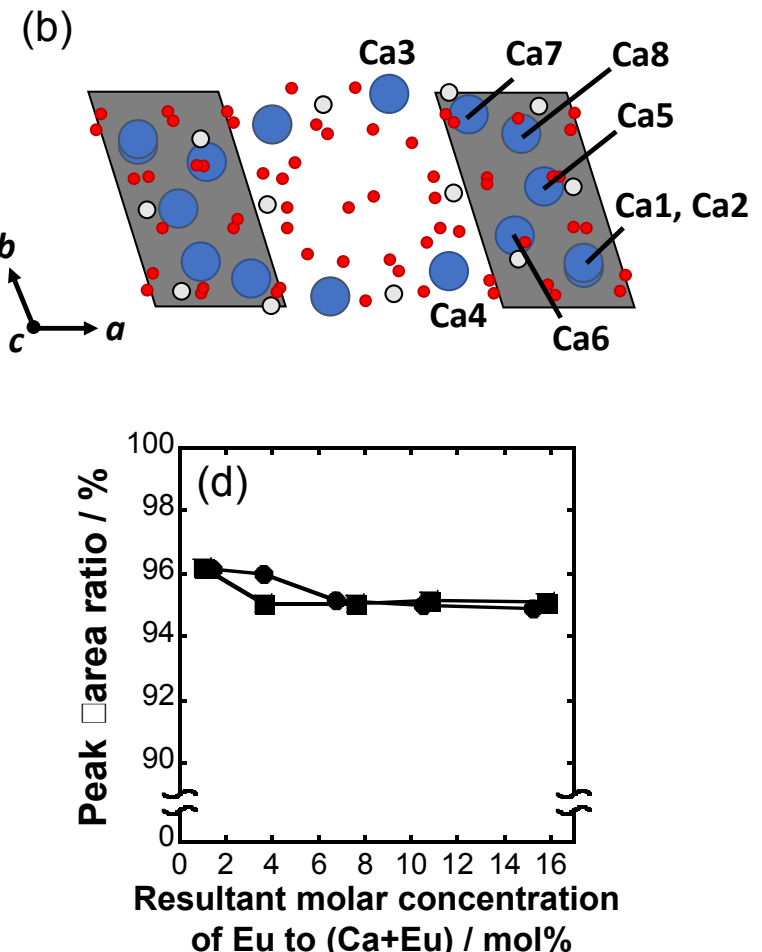

Figure S7. Illustration of Ca site position in the (a) HA and (b) OCP unit cell structure. (c) Representative deconvolution result of luminescence spectrum peak due to ${ }^{5} \mathrm{D}_{0} \rightarrow{ }^{7} \mathrm{~F}_{0}$ transition of Suc-OCP:Eu-10 for separating peak I and II under the excitation wavelength at $395 \mathrm{~nm}$. Here, the red and blue curves indicate the raw and re-synthesized spectra, respectively. (d) Separated peak I area ratio changes of $(\bullet)$ OCP:Eu-X and ( $\bullet$ ) Suc-OCP:Eu- $\boldsymbol{X}$ systems with the resultant molar concentration of Eu to $(\mathrm{Ca}+\mathrm{Eu})$. 
Figure S8

(a)

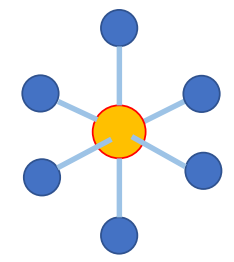

Octahedral complex (Centrosymmetry)

(b)

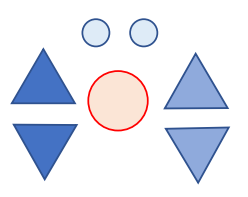

OCP:Eu

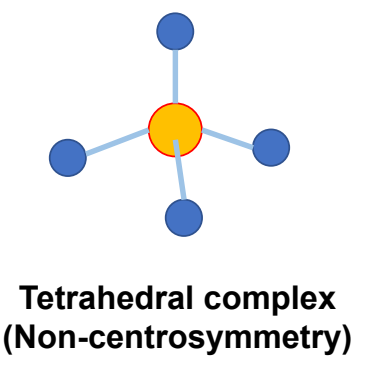

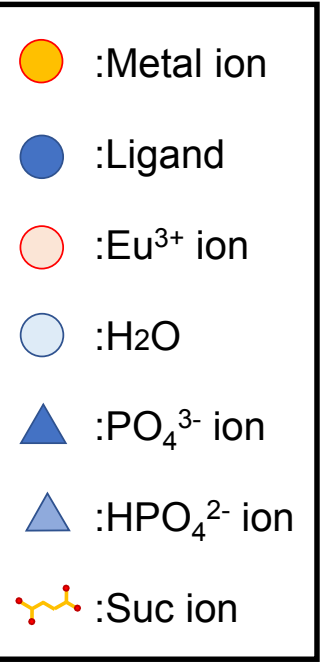

Suc-OCP:Eu

Figure S8. (a) Representative relationship between symmetry and luminescence intensity of metal complexes, and (b) their simplified illustrations of environmental symmetry of $\mathrm{Eu}^{3+}$ ion in the hydrated layer of OCP:Eu and Suc-OCP:Eu systems. 
Figure $\$ 9$
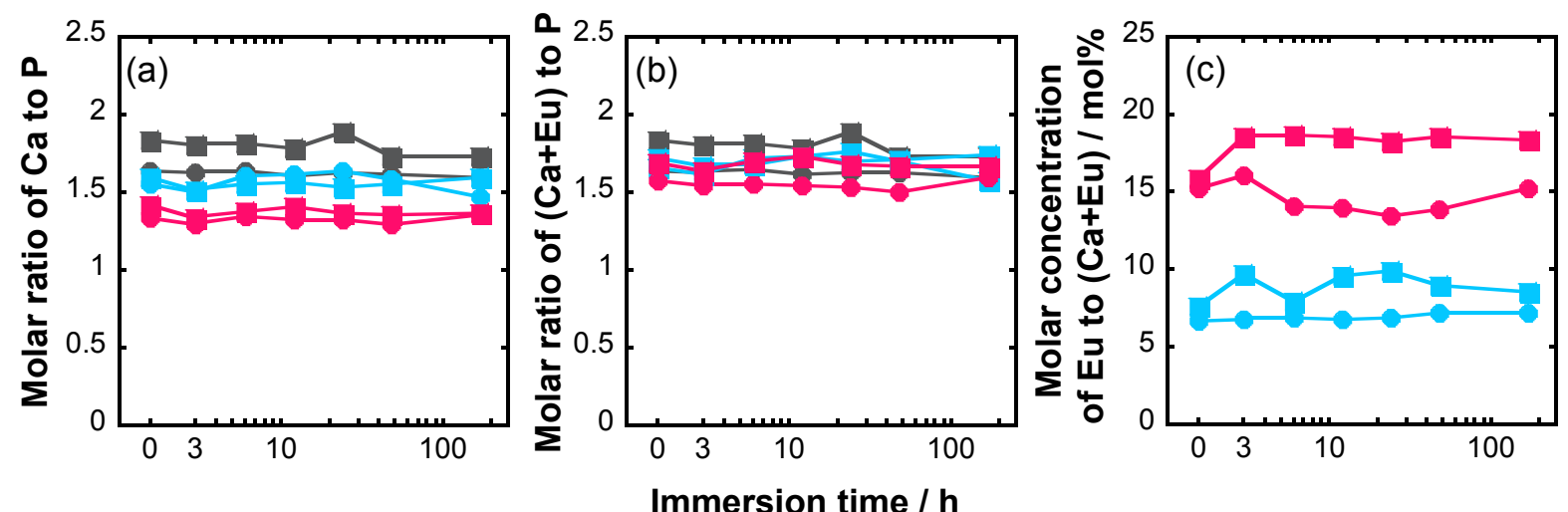

Figure S9. The changes in the molar ratios of (a) $\mathrm{Ca}$ to $\mathrm{P}$ and (b) $(\mathrm{Ca}+\mathrm{Eu})$ to $\mathrm{P}$, and (c) resultant molar concentration of Eu to (Ca+Eu) of OCP:Eu-X $(\boldsymbol{X}=\mathbf{0}(\bullet), 5(\bullet)$ and $10(\bullet))$ and Suc-OCP:Eu-X $(\boldsymbol{X}=\mathbf{0}(\boldsymbol{\bullet}), \mathbf{5}(\mathbf{\square})$ and $\mathbf{1 0}(\boldsymbol{\bullet}))$ with the immersion time in SBF, which were analyzed by the XRF. 
Figure S10

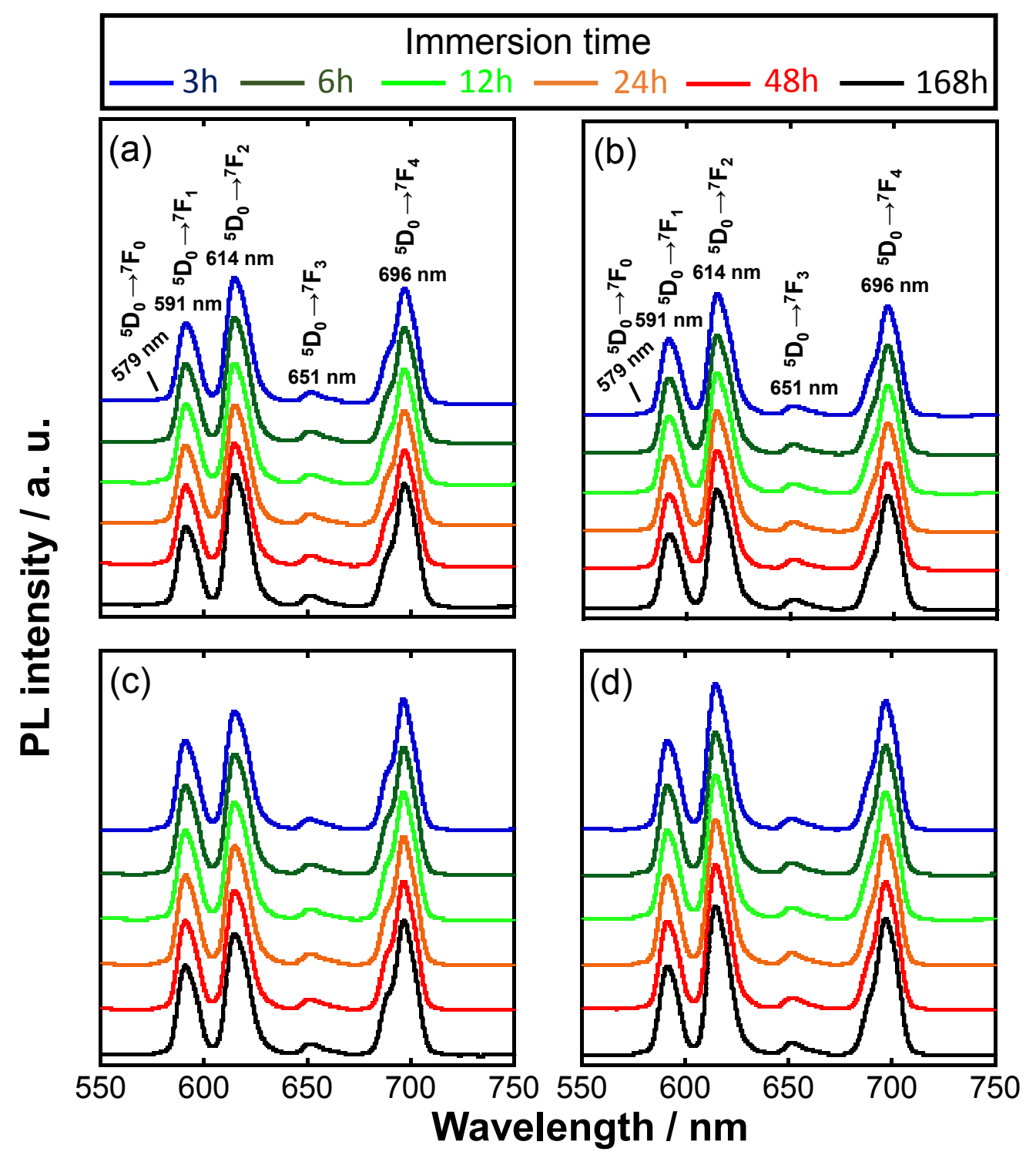

Figure S10. Luminescence spectra of (a) OCP:Eu-5, (b) OCP:Eu-10, (c) Suc-OCP:Eu-5 and (d) Suc-OCP:Eu-10 under the excitation wavelength at $395 \mathrm{~nm}$ at the different immersion times in SBF. 
Figure S11

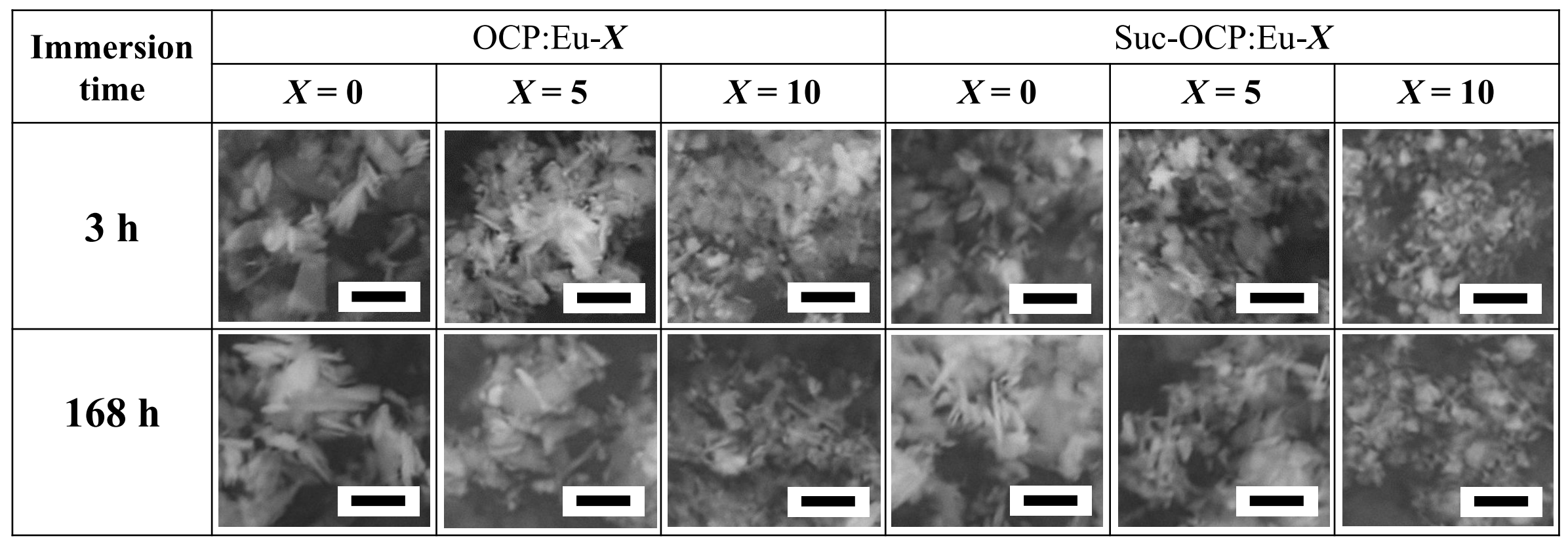

Figure S11. SEM images of OCP:Eu- $\boldsymbol{X}(\boldsymbol{X}=\mathbf{0}, \mathbf{5}$ and 10) and Suc-OCP:Eu- $\boldsymbol{X}(\boldsymbol{X}=\mathbf{0}, \mathbf{5}$ and 10) at the immersion times of $3 \mathrm{~h}$ and $168 \mathrm{~h}$ in SBF (scale bar: $1 \mu \mathrm{m}$ ). 
Figure S12

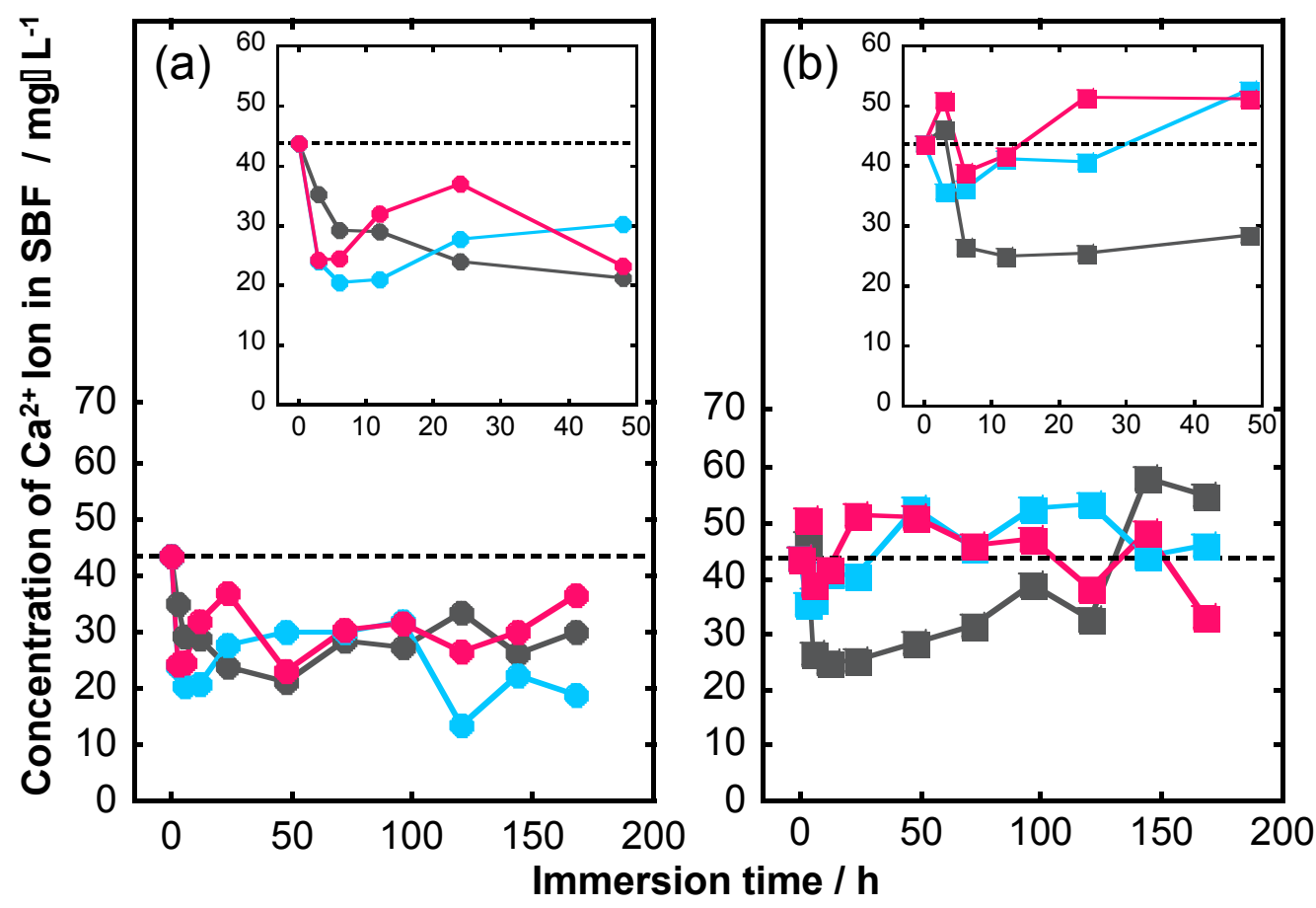

Figure S12. Concentration of $\mathrm{Ca}^{2+}$ ion in SBF with immersing (a) OCP:Eu- $\boldsymbol{X}(\boldsymbol{X}=\mathbf{0}(\bullet), \mathbf{5}(\bullet)$ and

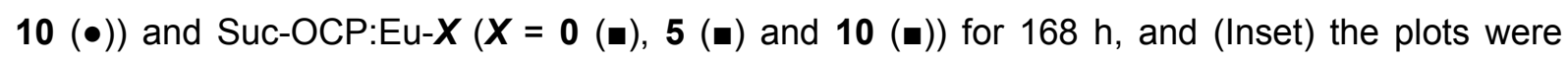
expanded for the short-term immersion for $48 \mathrm{~h}$. The dashed line indicates the initial $\mathrm{Ca}^{2+}$ ion concentration in SBF $(43.7 \mathrm{mg} / \mathrm{L})$. 
Figure S13

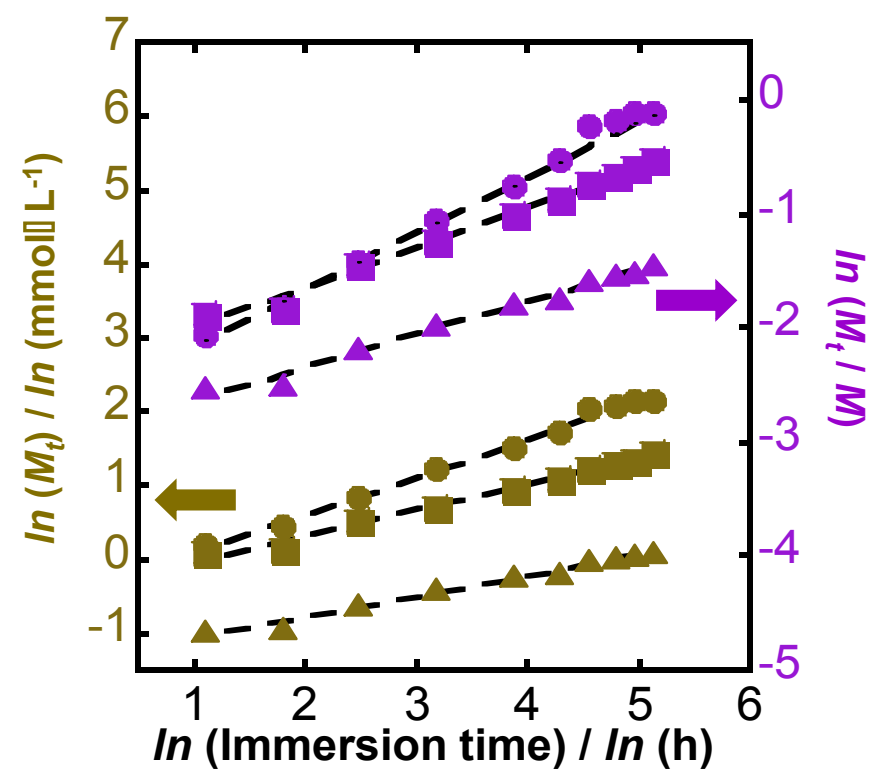

Figure S13. Korsmeyer-Peppas plots calculated from the $M_{t}$ and $M_{t} / M$ of the Suc-OCP:Eu- $\boldsymbol{X}(\boldsymbol{X}=$ $\mathbf{0}(\bullet$ and $\bullet), \mathbf{5}(\square$ and $\boldsymbol{\square})$ and $\mathbf{1 0}(\boldsymbol{\Delta}$ and $\Delta)$ ) with the immersion time in SBF. 


\section{Figure S14}

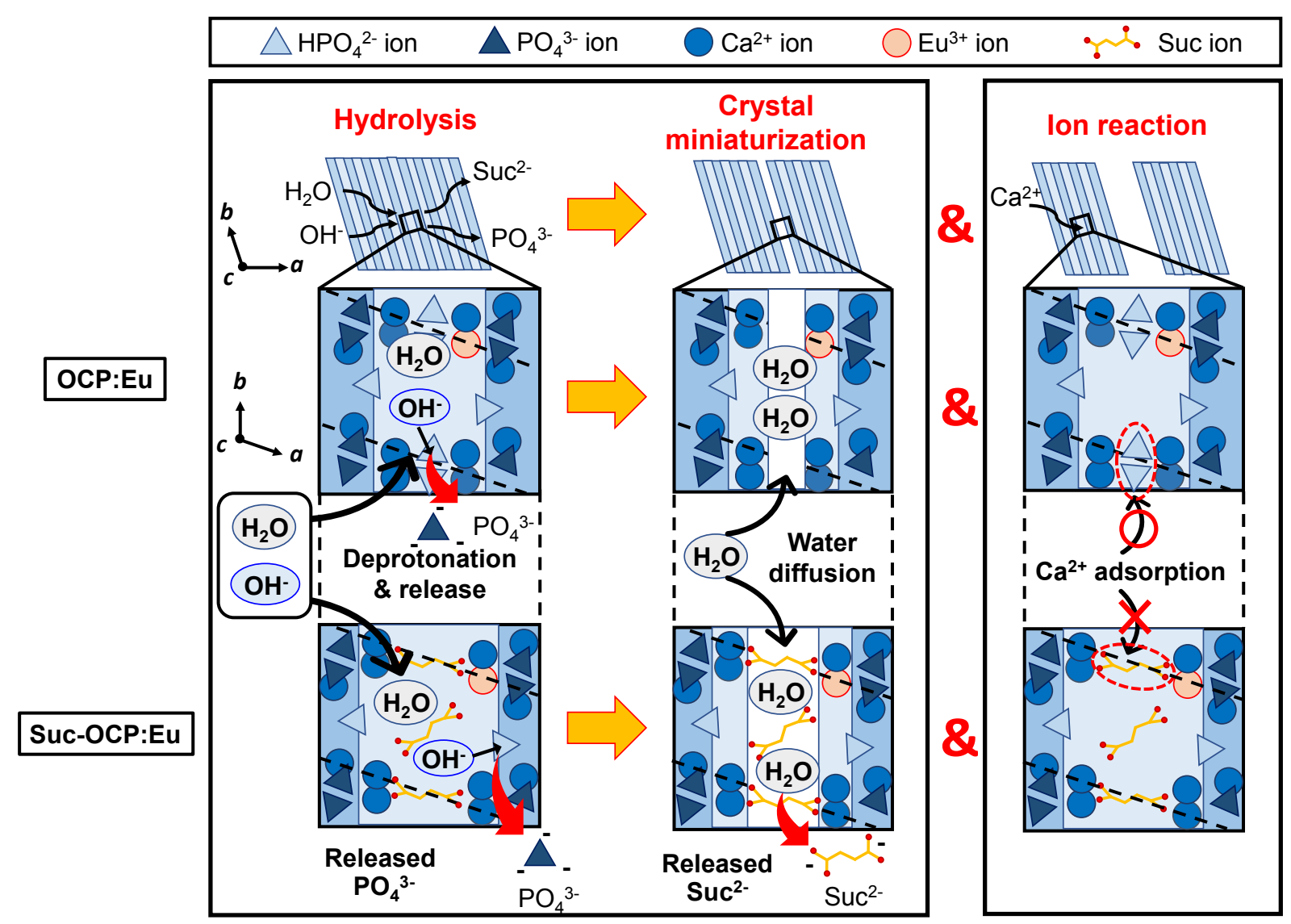

Figure S14. Illustration of representative interlayer functions (hydrolysis, crystal miniaturization and ion reaction) of OCP:Eu and Suc-OCP:Eu systems in SBF. 